\title{
Development of Correspondence Algorithm for Illumination Varying Dynamic Image Sequence using Symbolic Data Analysis: A Novel Approach
}

\author{
Y P Gowramma ${ }^{1}$, C N RaviKumar ${ }^{2}$ \\ ${ }^{1}$ Dept. of CSE, Kalpataru Institute of Tech, Tiptur-572202, Karnataka, India. \\ ${ }^{2}$ Dept of CSE, SJ college of Engineering, Mysore, Karnataka, India.
}

\begin{abstract}
This paper presents a novel symbolic data analysis based correspondence algorithm to identify the correspondence of illumination varying multiple object image sequence for the dynamic image analysis. The correspondence is the matching of the similar data sets or entities or the features such as the region based global features, points, corners, edges between a set of images. The Correspondence of illumination varying multispectral, temporal multiple objects using symbolic data analysis of objects in the dynamic images is a highly challenging problem. The determining Correspondence of features is a high dimensional NP-hard problem, because of their exhaustive search for the features in the sequence of image frames. The current algorithm works on the basis of successive frame difference based segmentation. The minimum boundary rectangle constructed for segmented moving object region. Mean of the rectangle is considered as one of the numeric feature. Next the sequence converted to binary sequence based on the threshold and 8-connectivity of perimeter is another non numeric feature. Finally establish the correspondence using canonical symbolic data analysis. This work proposes the rectangular correspondence window, generated dynamically which depends on the size and the number of objects in the current frame. Here we have considered mean of minimum boundary rectangle and perimeter of the segmented region as the features. The experiment is conducted with single object in the scene and multiple objects in the scene of illumination varying, noisy image sequence. This experiment is carried out on the binary, gray and RGB color dynamic image sequence. This correspondence algorithm is especially suitable for tracking in indoor images illuminated by unconstrained multiple light sources, objects in the varying illumination environment, noisy image sequence, video surveillance and slow moving dynamic image sequence for indoor scenes of the constant background.
\end{abstract}

Keywords: Successive Frame Difference, Symbolic data Analysis, Threshold Based Binary Conversion, Logical Image, Minimum boundary rectangle, 8Connectivity based Perimeter, Canonical Symbolic Correspondence.

\section{INTRODUCTION}

An Accurate, Automatic, Unconstrained, Optimized, Non initialized window based, Fast correspondence of features across two or more images is well known to be of crucial importance for many Computer Vision, Image Analysis and Pattern Recognition tasks. Reliable inter image feature correspondence and its closely related problems such as automatic object detection, human identification based on gait, automatic surveillance, that is monitoring a scene to detect suspicious activities or unlikely events. Further creating larger pictures, industrial applications, medical image processing, image registration, structure from stereo approaches, motion analysis, image mosaicing, 3D reconstruction, object recognition, stereo matching, feature and object tracking, video surveillance which involves the fundamental problem termed as the correspondence problem. Noisy, illumination varying, multiple object, and automatic correspondence is one of the challenging problem in surveillance and dynamic image analysis related problems.

The output of the correspondence algorithm is a set of tracks, where each track ideally corresponds to a unique point or an object in the dynamic image and specifies its position in every frame from entry to exit in the scene.

The complexity of the correspondence algorithm increases with (1) noise in images, (2) nonrigid or articulated nature of objects (3) Partial or full objects (4) scene illumination variations (5) Multiple objects in the scene.

Symbolic objects are extension of classical data types which are well adapted to represent the knowledge. In symbolic data analysis the objects are symbolic and they are usually combinations of numerical and nonnumerical data types. The description of symbolic objects may depend on the relations exiting between elementary objects, frequency of occurrence of the values and so on. In our proposed work we have applied symbolic data analysis concept for dynamic image analysis. The dynamic images are the temporal images. The RGB colour images are the multispectral images. The temporal multispectral data can be represented as symbolic objects of interval type and multivalued type. These are called quantitative and qualitative type of symbolic objects. 
Few of the researchers in the literature the satellite images are treated as the multi banded multispectral temporal symbolic data. In this images considered taken at half hourly interval and this data is considered as symbolic data. Here the symbolic data analysis concept is used for edge detection, clustering, classification of areas and management of natural resource using symbolic data analysis of temporal multispectral images. In our research work we have considered spatio temporal multi spectral data which has the colour dynamic image sequence. Spatio temporal multispectral images are the images captured at different time intervals, image pixels at every particular location may be considered multivalued. It could be tracking of moving objects in consecutive image frames. The time interval could be less than seconds to decades depending on application.

Video surveillance is the continuously recording field of view of the sensor. Here objects may enter and exits the scene in several possible ways. They enter from horizontal, vertical and diagonal direction and exit the scene in the any direction. The other possibility is partially enters and exits the scene in any direction. Here in the analysis we need to find the correspondence of the objects.

Illumination change is a natural phenomenon which occurs due to various reasons both on indoor and outdoor images. Outdoor illumination variation occurs due to natural light condition. Indoor illumination variation occurs due to artificial light i.e. the fluorescent light changes illumination at the frequency of power supply.

There is no general algorithm to solve all the correspondence problems. It is one of the difficult tasks in any of the dynamic image analysis problem. There are several algorithms proposed in the literature for correspondence under the highly constrained environment such as velocity smoothness, illumination constraints, noise free images, restriction on the number of moving objects, objects geometric transformation, degrees of freedom of the sensor. Establishing the automatic image correspondence in the unconstrained environment is ever green open active research challenge in computer vision.

In computer vision and pattern recognition, colour and texture are the important features for recognition. But during illumination varying environment and movement of the objects it is difficult to consider colour and texture because they change according to the light intensity. So the global region property perimeter connectivity of the features or pixels and the mean of the gray scale difference segment correspondence can be considered. For instance, this method can be applied to observe the movements of objects in the video surveillance.

In the last two decades, many fast correspondence methods have been proposed to give a faster intensity based correspondence algorithms. The proposed algorithm also automatically establishes the correspondence under photometric variations and geometric transformation such as scaling, transformation, rotation. The proposed work establishes the correspondence to track mean and 8-connectivity of perimeter features of the object in the dynamic image analysis.

We come across many research works on correspondence under constraint environment but there are only a few papers on illumination varying environment and no papers on symbolic data analysis in dynamic image analysis. There is no much literature on the correspondence for the illumination varying environment and entry and exit of objects. Here we present a sample of different methods proposed in the literature.

Helko Hirschmuller and Daniel[1] has evaluated various matching algorithms for stereo images with radiometric differences . But they considered only the gray scale images. Colour images are the out of scope for their research. They processed only the still images, but not dynamic images. SomaBiswas, Gaurav Aggarwal, Ramma Chellappa, [2] developed robust estimation of albedo for illumination Invariant matching and shape recovery for face images

Sethi and Jain [3] solve the correspondence by a greedy approach based on the proximity and rigidity constraints. Their algorithm considers two consecutive frames and is initialized by the nearest neighbor criterion. The correspondences are exchanged iteratively to minimize the cost. A modified version of the same algorithm which computes the correspondences in the back ward direction (from the last frame to the first frame) in addition to the forward direction is also analyzed. This method cannot handle occlusion, entries, or exits. Salari and Sethi [4] handle these problems, by first establishing correspondence for the detected points and then extending the tracking of the missing objects by adding number of hypothetical points. Rangaraj and Shah [5] propose a greedy approach, which is constrained by proximal uniformity. Initial correspondences are obtained by computing optical flow in the first two frames. The method does not address entry and exit of objects. If the number of detected points decreases, occlusion or misdetection is assumed. Occlusion is handled by establishing the correspondence for the detected objects in the current frame. For the remaining objects, position is predicted based on a constant velocity assumption.

Veenman et al. [6] extended the work of Sethi and Jain [1987], and Rangarajan and Shah [1991] by introducing the common motion constraint for correspondence. The common motion constraint provides a strong constraint for coherent tracking of points that lie on the same object; however, it is not suitable for points lying on isolated objects moving in different directions. The algorithm is initialized by generating the initial tracks using a two pass algorithm, and the cost function is minimized by Hungarian assignment algorithm in two consecutive frames. This approach can handle occlusion and misdetection errors; however, it is assumed that the number of objects is the same throughout the sequence, that is, no object entries or exits.

Chidanada gowda ana diday [7] presented unsupervised learning through symbolic clustering. Now we propose symbolic data analysis based novel algorithm for the correspondence algorithm for dynamic image analysis. 
This paper is organized in 7 sections. In section 2 the problem statement is detailed. The section 3 describes problem formulation and section 4 discusses assumptions, and constraints respectively. The section 5 describes the proposed methodology in detail. The Sections 6 and 7 presents the details of experiment analysis and conclusion.

\section{PROBLEM STATEMENT}

Image acquisition in the Dynamic images is considered from the following cases.

1. Static sensor and Moving objects in the scene 2 . Moving sensor and Static objects in the scene and 3. Both Sensor and Scene objects are moving.

Dynamic Images are also called as the sequence images. These images are the spatio temporal images. which may be also termed as sequence image analysis. Here we have considered the problem of finding the correspondence of features for the multiple objects in the image sequence frames $f_{1}, f_{2}, \ldots, f_{i}, \ldots \ldots, f_{n}$ at the distinct time instance $t_{1}, t_{2}, \ldots \ldots, t_{i}, t_{i+1}, \ldots \ldots, t_{n}$ respectively from the illumination varying environment of the indoor sequence. In this work we have proposed a novel symbolic data analysis solution methodology for the correspondence of tracking the objects in the RGB color, gray and binary image sequence under the illumination varying environment These frames are considered as the 2-D images. From these set of frame images $\mathrm{f} 1$ is considered as the reference image and the remaining image frames $f_{2}, f_{3} \ldots f_{n}$ are considered as search images. In addition to the above complexities, the solutions for the object leaving and reentering after many frames are being considered. It is invariant to ration and translation of the objects. It works efficiently for objects closer to the edge and corners during entry and exit. It also works for the partial and full objects in the scene.

\section{PROBLEM FORMULATION}

The images considered in this work are the monocular single moving sensor dynamic image sequence. The objects presented in the image frames are the slowly moving objects with varying velocities. The number of objects need not be constant through the sequence. As the intensity of the light varies under uncontrolled multiple light sources in each frame the intensity of the object also varies. Here the moving objects in the scene are not initialized for the correspondence. Long sequences of more than 1000 frames are considered for the analysis.

\section{CONSTRAINTS}

AND ASSUMPTIONS

The correspondence is made simple by imposing constraints on the motion and appearance of the objects. In this application we have tried to minimize the number of constraint on the motion and appearance of the objects. The only constraint on the motion of the object is that it should not make sudden change in the direction of motion while moving out of the viewing range of the camera. The object is little brighter than the background gives better tracking result.

\section{PROPOSED METHODOLOGY FOR CORRESPONDENCE}

The figure (1) shows the schematic diagram of the proposed correspondence approach for the flickering.

Input: The indoor color image sequence with the illumination varying by the multiple uncontrolled source of light.

Output: The correspondence of the objects in the consecutive frames shown as the rectangle window enclosing the object.

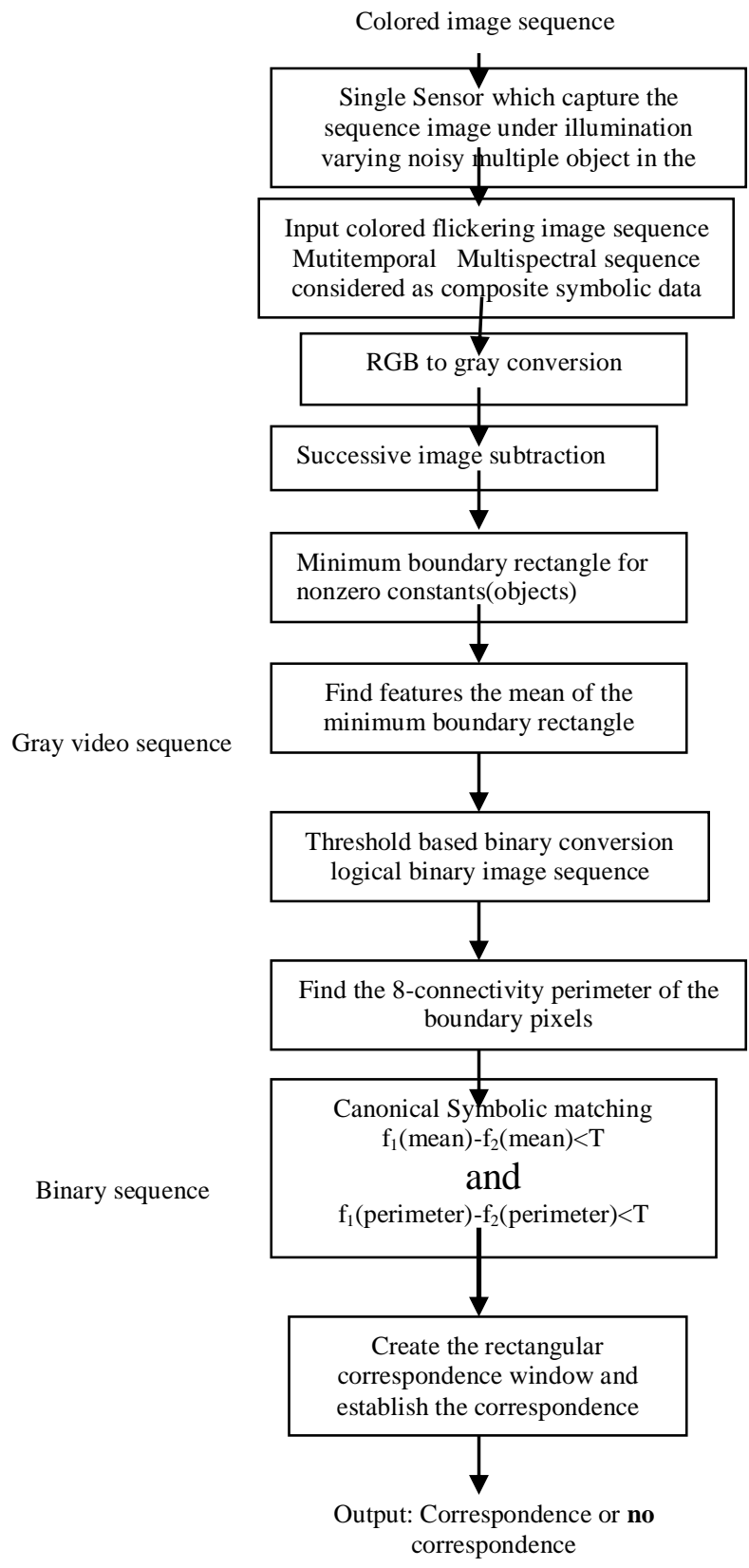

Figure 1.Schematic diagram of the proposed approach 


\subsection{IMAGE ACQUISITION}

A video camera capable of capturing the moving (dynamic) scene is used as the sensor. It will capture the image sequence in the RGB color format. There is no restriction on the illumination variation, smoothness of the velocity of the object, the velocity of the sensor and there is no restriction on the degree of freedom of the camera movements. The output of the image acquisition system is the RGB dynamic image frames under the illumination variation with the multiple objects present in the scene

The captured image sequence is in RGB color format at the rate of the 25 frame per second.

This RGB image sequence is converted into the grayscale sequence image. Since the RGB color format has 24-bits per pixel it takes more processing time compared to the gray scale image and binary image. The first frame is considered as the reference image by default. The construction of the reference image depends on the application and input sequence for accuracy and time complexity.

\subsection{Successive Frame difference based segmentation}

The long sequence dynamic images are segmented using successive frame difference. In the dynamic image analysis the various combination of the successive frame difference is used to segment the dynamic image. Let

$I_{d}(x, y)=\operatorname{absolute}\left(I_{c}(x, y)-I_{p}(x, y)\right)<T$

Where $\mathbf{I}_{\mathbf{c}}(\mathbf{x}, \mathbf{y})$ is the current frame, $\mathbf{I}_{\mathbf{p}}(\mathbf{x}, \mathbf{y})$ is the previous frame and finally $\mathbf{I}_{\mathbf{d}}(\mathbf{x}, \mathbf{y})$ is the difference frame. The difference frame is the combined information of the object movement and the sensor movement and the light source available with the sequence.

The difference between the consecutive frames is usually created by a combination of camera motion and the movement of the objects. The frame difference on consecutive frames in the image acquisition identifies moving objects from the portion of a video frame that differs significantly from the previous frame Here the difference frame sequence is stored separately and this sequence is used to convert the gray scale sequence to the binary image sequence. Then the threshold based conversion is considered.

\section{Threshold based Conversion$$
I_{d}(\mathbf{x}, \mathbf{y})>\mathbf{T}
$$$$
\text { bin_img }(\mathbf{j}, \mathbf{k})=\mathbf{1} \text {; }
$$$$
\text { else }
$$$$
\text { bin_img }(\mathbf{j}, \mathbf{k})=\mathbf{0} \text {; }
$$

We treat this binary image in the logical binary sequence useful to find the connectivity of pixels to find the objects.

\subsection{Minimum boundary rectangle}

A region can simply be described in terms of the smallest rectangle that encompasses it. For a binary image function $f(r, c)$ a region may be described as a set of points or pixels such that $S=\{(r, c) \mid f(r, c)=1\}$

Thus the smallest rectangle can be found in the simplest way find the connectivity of the object if it is upright. In that case, diagonally opposite corner points of the said rectangle are

$\left(\mathrm{r}_{\min }, \mathrm{c}_{\min }\right)$ and $\left(\mathrm{r}_{\max }, \mathrm{c}_{\max }\right)$, where

$r_{\min }=\min \{r \mid(r, c) \in S\}$

$\mathrm{r}_{\text {man }}=\operatorname{man}\{\mathrm{r} \mid(\mathrm{r}, \mathrm{c}) \in \mathrm{S}\}$

$\mathrm{c}_{\min }=\min \{\mathrm{r} \mid(\mathrm{r}, \mathrm{c}) \in \mathrm{S}\}$

$\mathrm{c}_{\operatorname{man}}=\operatorname{man}\{\mathrm{r} \mid(\mathrm{r}, \mathrm{c}) \in \mathrm{S}\}$

The minimum boundary upright rectangles may obtained by close approximation of minimum bounding rectangle when sides of the bounding rectangle are aligned with the principle axes of the region.

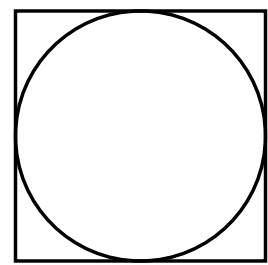

Figure 2. Shows the circle is bounded by the minimum bounded rectangle.

\subsection{Composite Symbolic Feature Extraction}

In the symbolic data analysis composite objects are formed as follows.

Let $\mathrm{A}$ and $\mathrm{B}$ are two features.

A having the features $\left(\mathrm{fea}_{1}, \mathrm{fea}_{2}, \ldots \ldots, \mathrm{fea}_{\mathrm{n}}\right)$

B having the features $\left(\mathrm{fea}_{1}, \mathrm{fea}_{2}, \ldots \ldots, \mathrm{fea}_{\mathrm{n}}\right)$

Here the intensity based statiscal numerical object features are mean, variance, mode, median, minimum, maximum,.etc. Feature based global features are area, connectedness, perimeter, centroid, entropy,., etc. are boolean features of the binary logical images are nonnumeric features. Any combination of these two forms the composite symbolic data feature.

\subsubsection{Mean of the difference image}

The first numeric feature is the mean of the result of the difference frame nonzero constants of the each regions bounded by the minimum boundary rectangles for the number of objects present in the image frame.

Mean $=1 / \operatorname{mn} \Sigma \Sigma \mathrm{f}(\mathrm{r}, \mathrm{c})$.

In this approach mean is one of the feature.

\subsubsection{Perimeter pixel connectivity}

The perimeter is boundary of 8-connctivity of the object of the binary segmented image. The perimeter is invariant to the rotation and transformation and illumination variations. This is the other nonnumeric Boolean region based global feature.

\subsubsection{Dimensionality reduction}

Dimensionality reduction is one of the important stage in any pattern recognition problem. Mean is the combination of the pixels intensities. The 8-connectivity 
of the perimeter is only the boundary of the $n$ pixels region. Hence the dimensionality is performed on the region containing $\mathrm{n}$ number of pixels.

Mean < n number of pixels

Perimeter connectivity $<\mathrm{m}$ number of pixels in the object region

Connectivity of the binary image pixels is one of the important concepts in the image analysis and object recognition. The two dimensional images derives the 4connectivity and the 8-connectivity .By default we consider the 8-connectivity to recognize the objects in 2 dimensional images. The number of pixels connectivity defined for any experiment depends on the size of object in the sequence.

\subsection{Finding the correspondence window boundary.}

In the detection of the boundary first convert the grayscale image into the binary image using the pixel threshold. The difference image is converted into the binary image.

In this stage the correspondence object boundary is detected. Here it starts growing in rectangular window which encloses the object wherever the connectivity is less than the specified pixel.

Here we have to draw the rectangular window about the boundary of the object in the gray scale and the RGB sequence. The window is constructed as and when it is required depending on the size of the object to enclose it.

\subsection{Canonical matching of Boolean symbolic objects}

In general, a Boolean symbolic object represents a class description (Diday 1990). Therefore, it can play the role of the referent in the matching process.

The canonical matching returns either 0 (failure) or 1 (success)

The values 1 and 0 according to

$\operatorname{Match}(\mathbf{a}, \mathbf{b})=\{$

$$
\begin{aligned}
& 1 \text { if } \mathrm{Bj} \leq \mathrm{Aj} \text { for each } \\
& \mathrm{j}=1,2, \ldots \ldots \mathrm{p} \\
& 0 \text { otherwise }
\end{aligned}
$$

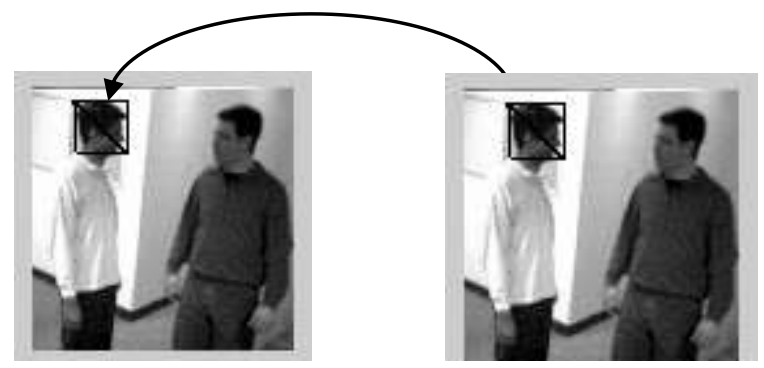

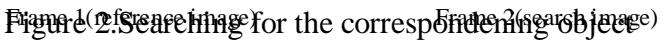

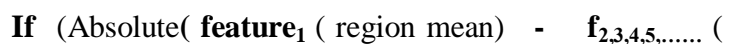
region 1 mean) )) < Threshold

And

(If Absolute( feature 2 (number of perimeter pixel 8connectivity) - $\mathbf{f}_{\mathbf{2}, \mathbf{3}, \mathbf{4}, \mathbf{5}, \ldots . . .}$ (number of perimeter pixel 8-connectivity) )) < Threshold

then

There is a correspondence of features(1)

else

No correspondence (0)

The correspondence is established based on the number of pixels connectivity of the perimeter in the consecutive images below the threshold and the mean of the region of the consecutive images below the threshold. The searching for the connectivity is 15 pixels in horizontal direction and vertical direction.

Algorithm

Step 1. Read the RGB color dynamic image sequence frame under illumination varying environment

Step 2. Identify the reference image

Step 3. Convert the RGB image sequence to the gray image sequence

Step 4. Apply the successive image subtraction.

Step 5. Construct minimum boundary rectangle which enclose the segmented moving objects and find the mean of minimum boundary rectangle.

Step 6. If the difference in gray value is greater than the threshold is considered as foreground else it is considered as background.

Step 7. Convert the binary sequence image into the logical image which is used to find the connectivity.

Step 8. Find the perimeter 8-connectedness of each image frame.

Step 9. Find the number of objects in the scene which has less than $\mathrm{n}$ pixels connectivity.

Step 10. Find any new object enters or exists the scene.

Step 11. Construct the rectangular window correspondence boundary and when required depends on the size, appearance of the object and the connectedness of the object and mean of the region.

Step 12. The correspondence windows are generated as and when the appearance of the object changes. Hence the object correspondence establishes the consecutive frames.

\section{EXPERIMENTAL ANALYSIS}

In this work the sequence image frames considered for the experiment are captured by mega pixels camera at the rate of 25 frames per second. The object undergoes illumination variation by the uncontrolled natural and 
artificial illuminations in indoor image sequence. The video is indoor sequence of the constant background, captured on a stationary camera.

The correspondence process is shown in separate windows of different types of images such as color image, gray-scale image, and binary images with different frames. The present experimental sequence consists of more than 500 frames. This experiment has six indoor sequences. The sequences having two objects with varying illumination and the algorithm which track the sequence will find the correspondence It works accurately for any sequence image which undergoes motion due to sensor movement or object movement where the objects are little brighter than the background. The object correspondence for two objects are enclosed in rectangular windows in the figure (3). The table one explains the summary of results.. The algorithm corresponds for all the objects in the rectangular window.
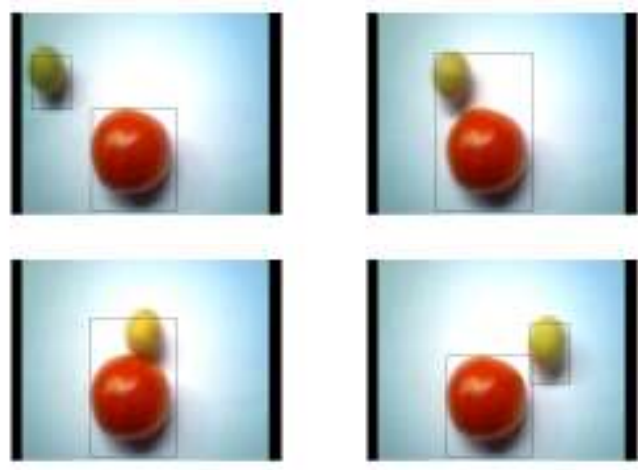

Row $1 \mathrm{a}$ b c d Object entering from left and leaving the right boundary with overlap

Figure (3) Entry and Exit of object in various stages under illumination varying environment

\section{Table1 summary of results}

\begin{tabular}{|l|l|l|}
\hline $\begin{array}{l}\text { Frame } \\
\text { number }\end{array}$ & $\begin{array}{l}\text { Execution } \\
\text { time }\end{array}$ & $\begin{array}{l}\mathrm{X}, \mathrm{Y} \text { coordinates of } \\
\text { object antidiagonal } \\
\text { corners }\end{array}$ \\
\hline 124 & 0.2500 & $\begin{array}{l}(120,213)(196,114) \\
(234,403)(395,215)\end{array}$ \\
\hline 128 & 0.1406 & $(211,404)(395,215)$ \\
\hline 130 & 0.1406 & $(232,404)(192,141)$ \\
\hline 132 & 0.2656 & $\begin{array}{l}(388,270)(463,152) \\
(232,404)(393,214)\end{array}$ \\
\hline
\end{tabular}

\section{CONCLUSION}

In this Paper, we have successfully proposed the correspondence of dynamic images for the moving objects in the illumination varying environment .The proposed algorithm locate the features of the reference frame to the search frame. The experiment is conducted on the binary image sequence; gray scale image sequence and RGB color image sequence in which the sequence undergoes slow moving indoor sequence which is captured by the static sensor and moving object. Hence we can conclude that our algorithm satisfactorily locate the enter and exit of moving object features in a consecutive image frames in the illumination varying environment. It works for multiple objects. The number of objects need not be constant throughout the scene. The velocity of the objects need not be constant. It is invariant to rotation and translation of objects. The window created to establish the correspondence of objects, dynamically adopts the size and shape of the objects in the scene. The window moves along with the objects and it appear and disappear in the scene in accordance with the appearance and disappearance of the moving objects in the scene. This method is suitable for image sequences having slow motion and tracking of any objects in the sequence which has motion.

\section{REFERENCES}

[1] Helko Hirschmuller and Daniel," Evaluation of Stereo Matching Costs on Images with Radiometric Differences",IEEE on Pattn.Analysis and Machine Inttegence,pp 1582-1598,vol.31,no.9,September 2009

[2] SomaBiswas, Gaurav Aggarwal, Ramma Chellappa, "Robust Estimation of Albedo for Illumination-Invariant Matching and Shape Recovery" ",IEEE on Pattn.Analysis and Machine Inttegence,pp 884899,vol.31,no.5,May 2009

[3] Rangarajan .K and Shait.M., 1991, establishing the motion correspondence, Conference Vision Graphics Image processing 54,1,56-73.

[4] Sethi .I.and Jian .R. 1987 ,Finding trajectories of feature points in a monocular image sequences, IEEE transactions on Pattern Analysis and Mach.Intell 9,1,5673 .

[5] Salari .V and Sethi I.K.1990 ,Feature point correspondence in the presence of occlusion ,IEEE Trans. Patt.Analysis.Mach.Intell.,12,1,87-91.

[6]Veenman.c.,Reinders.M. and Backer.E.2001.Resolving motion correspondence for denly moving points,IEEE Trans.Patt.Analysis Mach.Intelli,23,1,54-72

[7] K. Chidananda Gowda, E. Diday, "Unsupervised learning through symbolic clustering", Pattern Recognition Letters, Vol.12, pp. 259-264,1991.

[8] Gowda.K.C and E.Diday(1991). Symbolic clustering using a new dissimilarity measure.Pattern Recognition 24(6).

[9] Diday.E. (1993). Data to knowledge, boolean, probabilistic, possibilist and belief objects for symbolic data analysis. Tutorial at IV conf.IFCS.

[10] K.Chidananda Gowda and E.Diday,"Symbolic clustering using a new similarity measure", IEEE Trans. On systems man and cybernetics, Vol. 22, No.2,MarchApril 1992. 